Images du travail, travail des images

$2 \mid 2016$

Les ouvriers et la photographie : de 1945 à nos jours

\title{
Le documentaire critique et la représentation du travail ouvrier
}

Critical realism and images of work

Géraldine Millo

\section{OpenEdition}

Journals

Édition électronique

URL : https://journals.openedition.org/itti/1170

DOI : 10.4000/itti. 1170

Éditeur

Université de Poitiers

Référence électronique

Géraldine Millo, « Le documentaire critique et la représentation du travail ouvrier », Images du travail,

travail des images [En ligne], 2 | 2016, mis en ligne le 01 septembre 2016, consulté le 23 juillet 2021.

URL : http://journals.openedition.org/itti/1170 ; DOI : https://doi.org/10.4000/itti.1170

Ce document a été généré automatiquement le 23 juillet 2021.

Images du travail, travail des images 


\title{
Le documentaire critique et la représentation du travail ouvrier
}

\author{
Critical realism and images of work
}

\section{Géraldine Millo}

1 La dimension documentaire de la photographie offre au chercheur ainsi qu'au grand public des éléments de découverte et de compréhension de ce qu'ont été et de ce que sont la classe ouvrière et le travail ouvrier. Cependant, une certaine photographie documentaire contemporaine impose d'être interrogée avec les outils de la théorie de l'art et de l'esthétique, tout autant que ceux de l'histoire et de la sociologie dans la mesure où elle fonctionne sur un principe de transdisciplinarité, les photographes convoquant eux-mêmes les méthodologies scientifiques au profit de la création documentaire. Cette photographie documentaire ne vise pas tant à constituer un témoignage sur des réalités qu'à produire des dispositifs de recherche et de réflexion. Dans l'histoire de l'art, elle s'inscrit autant dans la question du style documentaire formulé par Walker Evans, mais mise d'abord en œuvre par August Sander que dans les pratiques productivistes des avant-gardes progressistes. Cette double origine souligne d'une part son souci de l'esthétique et d'autre part son engagement politique. Le souci de l'esthétique ne doit pas être entendu comme un désir de faire beau ou de créer une belle composition, mais de produire une esthétique efficace. L'efficacité documentaire a trouvé différentes formulations, dans le photomontage notamment au moment des luttes anti-capitaliste, anti-fasciste ou anti-impérialiste. Mais la photographie documentaire qui nous donne à voir le travail ouvrier dans ses problématiques contemporaines s'inscrit davantage dans la voie ouverte par le français Eugène Atget et l'allemand August Sander, et d'une certaine manière dans le courant littéraire réaliste du XIX siècle. Dans cette pratique de l'image, la mise à distance du réel et l'étude des ramifications et structures du sujet photographié sont prégnantes. C'est pourquoi cette pratique est souvent plus proche des méthodologies des sciences humaines que du photoreportage. L'engagement y est d'autant plus intense que le photographe tentera de construire des axes de compréhension plutôt que d'empathie, en appellera aux capacités de pensée et de lecture du spectateur plutôt qu'à ses capacités de compassion. 
Un tel mouvement photographique n'est compréhensible que dans la mesure où il est contextualisé dans notre actualité. En effet, photographier la classe ouvrière aujourd'hui impose de comprendre les mutations dans lesquelles elle est prise, pour ne pas reproduire à l'infini les poncifs construits sur une cristallisation de la grande époque, poncifs ayant plus ou moins de pertinence et de vérité appliqués à la situation contemporaine et/ou transportés à l'étranger dans des pays en développement. Bien que la classe ouvrière n'ait jamais été aussi unifiée que les images et la littérature ont bien voulu le montrer, cette prétendue unité est aujourd'hui plus que remise en cause, en même temps qu'elle est élargie sous l'appellation plus vaste et encore plus imprécise de classe populaire. Loin de fournir un témoignage, la photographie documentaire critique construit son propre système d'approche, de compréhension et de restitution et nous invite à penser le travail ouvrier dans le contexte du néocapitalisme.

Plutôt que de définir in abstracto cette photographie, nous proposons de parcourir au travers de trois œuvres ce que cette photographie a à dire et à montrer du travail ouvrier actuel et les moyens que les photographes mettent en œuvre pour le faire. Le choix de présenter aussi un travail documentaire que j'ai réalisé est justifié par l'intention d'interdisciplinarité qui meut le mouvement de la photographie documentaire contemporaine, dans lequel mon propre travail théorique et plastique s'inscrit. Trois axes sont ainsi présentés : le travail d'Allan Sekula aborde la question du travail ouvrier maritime par une recherche sur les formes mondialisées du transport maritime ; celui de Philippe Bazin met en perspective la notion de travail dans sa forme dominée. Dans ces deux travaux la question de la visibilité du travail ouvrier est chaque fois au cœur du sujet: Sekula nomme son documentaire filmique The forgotten Space, complément de son travail photographique, et insiste sur l'isolement et l'invisibilité des travailleurs du port, l'œuvre de Bazin est toute entière tournée vers la mise en cause des réalités que l'on occulte. Enfin, mon propre travail vise à interroger ce que devient l'école des ouvriers dans le contexte actuel et à montrer ces jeunes inscrits dans de tels parcours.

\section{L'isolement et l'invisibilité de l'ouvrier maritime : Allan Sekula}


Photographie 1. Allan Sekula, Des tuyauteurs terminent la salle des machines d'un thonier. Chantiers navals Campbell. Port de San Diego, août 1990.

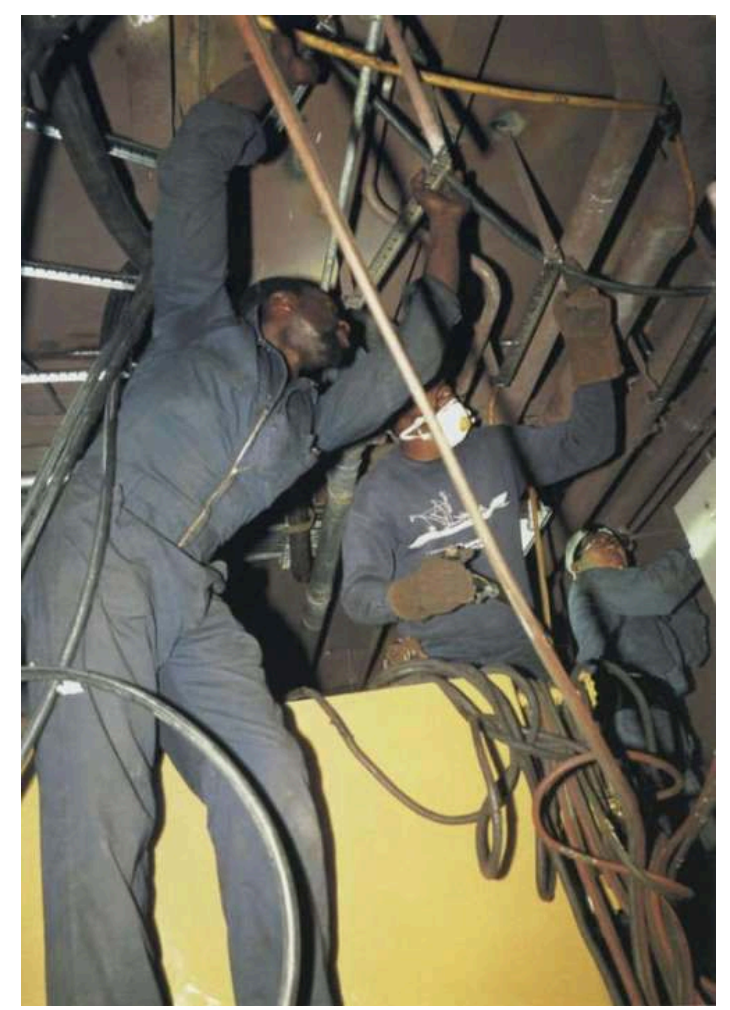

3 La photographie documentaire critique dont le photographe Allan Sekula est l'un des initiateurs organise autour des images un discours critique visant à dénoncer les processus de domination et d'aliénation et à produire une œuvre qui ne soit ni avec ni sur ni contre, mais qui mettent en perspective et explique les tensions et les mécanismes politiques menant à l'inégalité sociale et à son corollaire, la domination. La photographie documentaire critique prend le contre-pied d'une photographie se focalisant sur les ouvriers pour aborder le contexte plus large dans lequel les problèmes de la classe ouvrière ont leur source. Ainsi, bien que la question du travail et du travail ouvrier dans les ports et le transport maritime soit au cœur du travail de Sekula, ce qui est abordé dans son travail c'est le contexte global de la mondialisation et de la rationalisation des échanges. Les photographies montrant des ouvriers au travail sont ainsi prises dans un réseau d'images figurant tout autre chose : des espaces portuaires reconvertis en plateaux de tournage, des maquettes de bateau, des mobile homes déplacés sur des camions... Ce qui est fondamental dans ce type de travaux documentaires, c'est l'alliance du travail sur l'image et de la recherche théorique. Cette approche a un présupposé matérialiste chez Sekula : elle postule que les événements formels et symboliques s'appuient sur la forme de la production et impose donc d'interroger et de montrer celle-ci. Dans ce sens, Allan Sekula relève les signes visibles de cette organisation, les rassemble et les interroge : "plus le mouvement des biens dans les ports est régularisé, véritablement conteneurisé, autrement dit plus il est rationalisé et automatisé, et plus le port ressemble à la bourse. » (Sekula, 1995, 4)

Allan Sekula précise que Fish Story est le troisième volet d'un "cycle consacré aux géographies imaginaires et matérielles du capitalisme avancé » (Sekula, 1995, 202). La mise en cause de la rationalisation des échanges, l'étude de sa part d'idéologie et des impacts de celle-ci sur les hommes et leur environnement, qui font l'objet de Fish 
Story, sont répétés dans la structure même de son œuvre. Le photographe n'adopte pas une narration classique ni n'emprunte la forme classique du document, mais fait jouer les capacités descriptives de la photographie de telle sorte qu'elles prélèvent des fragments de réel qui sont les indices de la réalité imaginaire et concrète du capitalisme avancé. Chaque image n'agit pas comme une réalité pleine, de l'ordre du fait-objet, mais au contraire comme une trace partielle, signe dynamique qui renvoie à une structuration qui n'est pas en elle-même visible. L'indice n'exprime pas l'objet mais y fait référence : il n'a pas son caractère plein, mais propose une logique de la liaison plus que de la monstration. Cette manière de traiter l'image est adéquate à la méthode mise en place par Sekula et caractéristique de la photographie documentaire contemporaine. De même, le métissage entre des textes de différents niveaux (théorique, poétique, journalistique...) et des images de différentes sources (photographie, vidéo, film, archives, dessin...) caractérise à la fois le travail de Sekula et celui des artistes documentaristes. Ainsi, Sekula convoque autant le langage poétique, que le langage discursif, met au travail les différentes manières d'user du langage, symptômes de différents niveaux d'utilisation du réel. L'image est en besogne dans les mêmes directions : elle ne constitue pas une documentation à part entière, une documentation claire et classique des apparences du milieu maritime et du travail qu'il implique. Les images circulent dans les différents niveaux de réalité, dans les réalités imbriquées les unes dans les autres. C'est à une architecture-réseau que Fish Story renvoie, plutôt qu'au développement linéaire d'une narration orientée. En cela, la structure de l'œuvre mime la complexité du réel dans laquelle est pris le travail ouvrier. Alors que la cacophonie des échanges marchands faisait des ports des lieux de vie, de débauches, d'excentricités, de sueurs et de saveurs, la rationalisation du traitement des marchandises, imposée par la complexification, l'intensification et la mondialisation des échanges, a transformé radicalement les zones portuaires et son travail ouvrier. La marchandise circule sans transpirer, anonyme et les travailleurs se retrouvent isolés, peu nombreux et coupés de la ville. Face à cette situation, l'enquête ne peut pas aller d'un point $\mathrm{A}$ à un point $\mathrm{B}$ de manière linéaire : elle est parcours, processus en cours d'évolution, recherche des articulations, des points de visibilité qui permettront de comprendre les réalités du travail maritime et ses enjeux. Elle ne se concentre pas sur la description d'une situation à un endroit et temps donnés, mais sur les liens qui l'unissent à d'autres situations et d'autres lieux. Rendre visible les enjeux de la mondialisation, est ce à quoi s'attache une telle méthode et de telles images.

5 Le réalisme des images est ainsi très complexe car il parcourt moins un ensemble visuellement cohérent, que des lignes de force qui relient des réalités éparses entre elles. Qu'ont en effet à voir ensemble dans une même série les vestiges d'un plateau de tournage sur un chantier naval désaffecté et un quartier coréen à Los Angeles? Le réalisme de ces images articule de manière critique ce que le texte analyse : «L'espace se transforme. Les profondeurs marines sont sonorisées. Des bateaux de pêche disparaissent dans la mer d'Irlande, précipités au fond par des sous-marins. Les hommes d'affaires dans leurs avions lisent des romans passionnants où il est question de sonars. On démolit les bordels sur les quais ou on les rénove pour en faire des appartements. On convertit des chantiers navals en plateaux de tournage. » Ce n'est donc pas un ensemble dont la cohérence est à rechercher dans une continuité visible : le propre du réel est d'être éclaté aux quatre coins du globe et la réalité des ports d'être masquée par sa mise en boite et sa mise à l'écart des villes. Ainsi l'illusion qu'avaient les habitants des ports de pouvoir voir l'économie mondiale défiler sous leurs yeux se 
trouve remplacée par un contenu anonyme caché dans les conteneurs. De même, les bateaux arrivant ne sont plus les reflets de puissances économiques et politiques, mais des groupements de pays, dont l'un possède le pavillon, l'autre la marchandise, encore un autre l'équipage et enfin un dernier le capitaine. Enfin, les ports se trouvent pris entre un imaginaire de la mer et la réalité d'échanges commerciaux: "L'ancienne façade de port, que le chômage a coupé de ses liens avec un fond culturel commun, passe au service d'une rêverie bourgeoise sur le passé mercantile.» Les chantiers navals se trouvent effectivement recyclés en plateau de tournage, en même temps que les docks se trouvent transformés en appartement ou centres commerciaux, avec vue sur la mer. "Tout le monde veut voir la mer. » Les images confrontent ainsi ces réalités visibles entre elles : la manière dont elles n'ont rien à faire ensemble, mais se trouvent indéfectiblement liées, révèlent les rapports de forces qui se jouent dans le façonnage de la réalité visible des ports et ce qui les sous-tend, la structuration économique du capitalisme avancé.

Photographie 2 et 3 . Allan Sekula, Le chargement des cuves de l'aciérie Kaiser sur le vraquier Atlantic Queen en partance pour la Chine. Port de Los Angeles. Wilmington, juillet 1994.

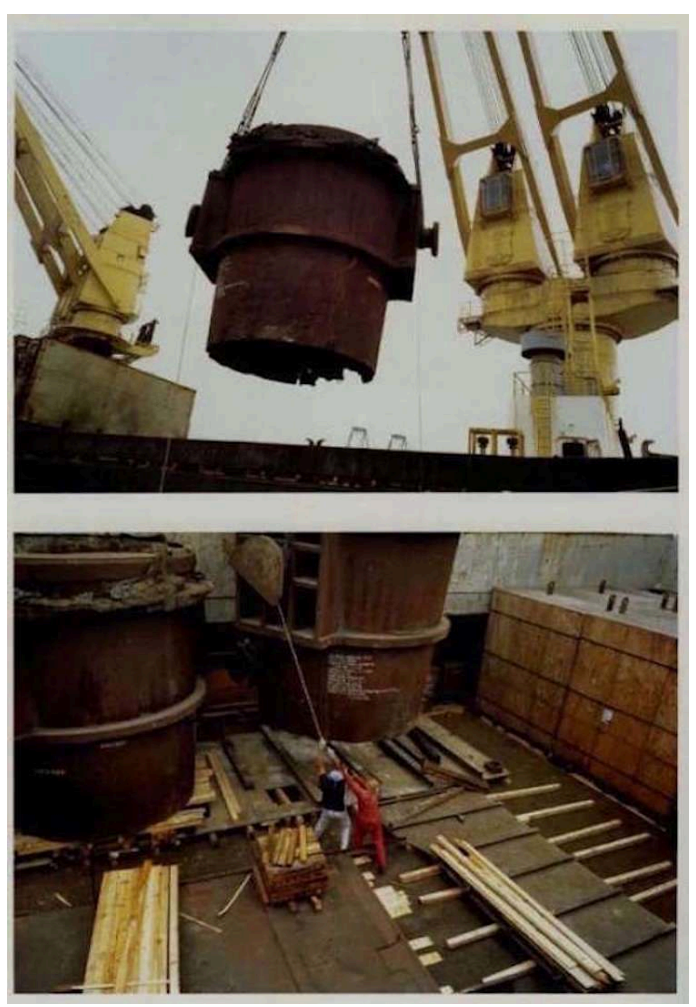

Les deux photographies présentées ici appartiennent au chapitre « la dictature des sept mers ». Elles font partie d'une série de cinq images autour de l'aciérie Kaiser. La force des analyses de Sekula réside dans sa capacité à mettre en scène, à donner à voir et à entendre, les histoires qui fabriquent le réel. Ainsi, la dictature des sept mers est-elle le symbole du rêve anarchiste de construire une solidarité transnationale des travailleurs de la mer : « une république ouvrière des sept mers, une vaste conspiration syndicaliste informelle de travailleurs des navires de commerce, des docks et des bateaux de pêche, reliant un port à l'autre avec son mépris itinérant pour les drapeaux et frontières. » (Sekula, 1995, 183) Sekula n'illustre pas cette histoire par des photographies : l'histoire fonctionne en marge du récit photographique. Ce sont des récits qui s'appareillent aux 
photographies, et réciproquement. Cette manière d'engager les images par rapport au texte correspond à l'étude du devenir des faits, des organisations que Sekula mène : les images montrent un devenir de la vision, travaillent sur les plaques qui relient des visions précédentes à des visions actuelles; les textes aussi parcourent ces lignes d'histoire qui s'imbriquent. « les belles résidences japonisantes des frères Greene et les plus modestes pavillons Craftsman du vieux Pasadena et du centre de Los Angeles furent bâties avec du bois coupé et transporté par des anarchistes. Quand on a rasé cette génération de logements dans les années quatre-vingt (...) le bois a suivi l'ancien itinéraire des anarchistes californiens remuants, partis rejoindre les frères révolutionnaires Flores Magon à Tijuana en 1911 pour édifier une république ouvrière éphémère. À présent, Tijuana prend ce que Los Angeles jette. »

7 L'approche critique de Sekula, qui déstructure une narration trop systématique et déstabilise la forme du document, installe ainsi le réalisme, non pas dans la perspective d'un doublage conventionnel du réel, mais dans une dynamique d'enquête face à celuici. D'une certaine manière, même s'il parie sur le fait que le visible est apte à fournir des indices pour comprendre le monde contemporain, l'enquête réfute l'idée d'une visibilité pleine : c'est pourquoi elle ne peut être que fragmentaire. L'incompréhension visuelle de la série d'images n'appelle que mieux le spectateur à créer pour lui-même cette géographie mentale qu'elle parcourt. Des images aux textes, des photographies aux légendes, le spectateur peut alors construire lui-même l'enquête au lieu de la consommer. Dans l'ouvrage consacré au travail d'Allan Sekula, Critical realism. Around Allan Sekula, Hilde von Gelder et Jan Baetens relèvent que le réalisme contemporain n'est plus reproducteur mais producteur (von Gelder, Baetens, 2007). S'il est passé du rapport mimétique au réel à celui de productif, c'est parce qu'il a évolué d'un rapport de transparence avec la réalité visible à une élaboration critique de celle-ci. Le réalisme ne cherche plus à imiter pour faire saisir le sens, mais à recomposer le réel pour révéler la part indiciaire des surfaces visibles. Les conventions que portent le réalisme témoignent d'une manière de rendre la réalité : le réalisme critique s'interroge sur ses moyens. La question que pose Sekula est: «quel sens cela a-t-il de représenter les choses de cette manière dans ce contexte?» Le style n'est alors pas un surplus esthétique mais un outil d'interrogation. La forme documentaire est ainsi mise en abîme: posée comme forme, elle est interrogée dans le processus même de son déroulement. Les fragments visuels que proposent ainsi Sekula s'emboitent aux écrits qui les accompagnent pour construire ce que Hilde von Gelder nomme « un montage des frontières ».

\title{
2. Voir le travail : Philippe Bazin
}

\author{
« Une forme documentaire travaille sur les choses \\ qui sont sous-jacentes à l'évènement médiatique. \\ Quelque chose qui est une lame de fond et qui \\ n'est pas dans les apparences. » \\ Philippe Bazin ${ }^{2}$
}


Photographie 4. Philippe Bazin, Dufftown 9. Une heure de travail, 2002. 8 vidéos de 60'.

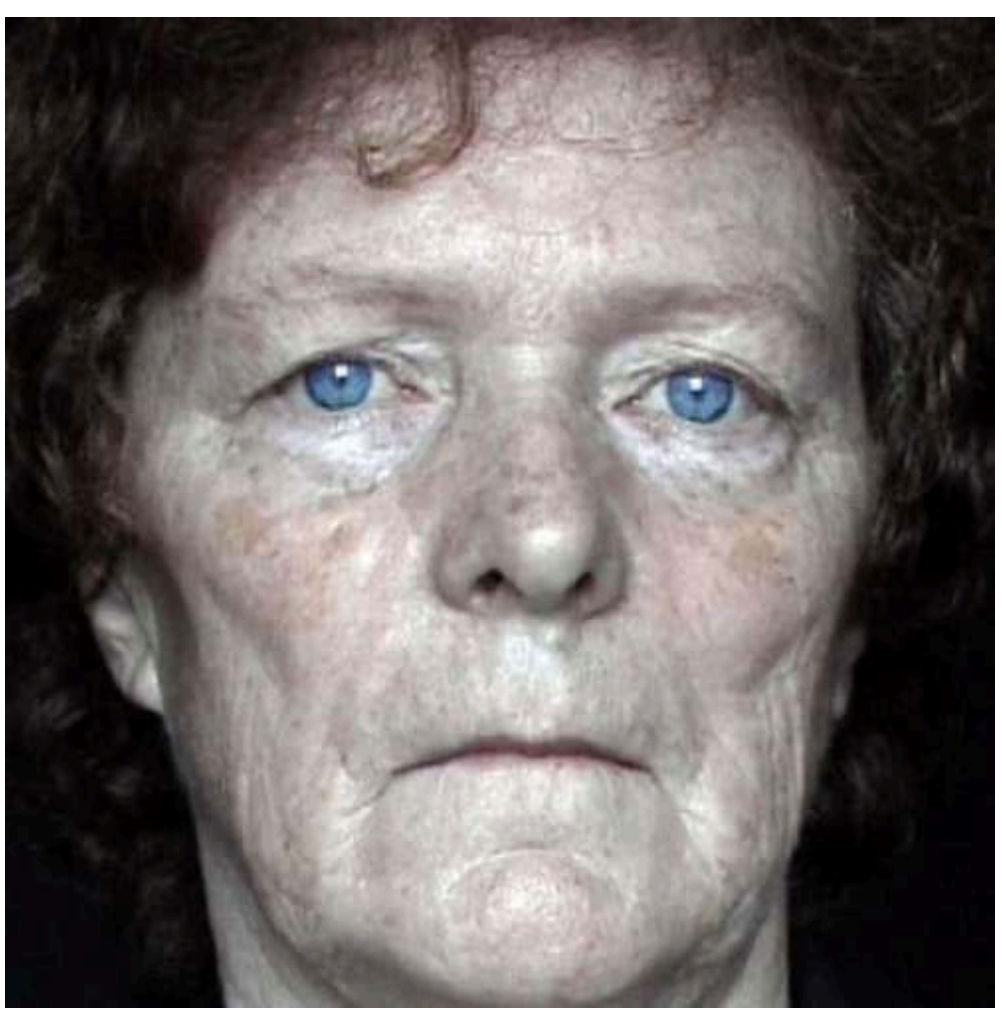

8 Un autre dispositif visant à parler du travail ouvrier est celui proposé par le photographe Philippe Bazin au travers de vidéos d'une heure intitulées Dufftown. Philippe Bazin ne procède pas là par enquête mais formule une forme problématique. Il ne montre pas le travail ouvrier ni n'aborde son contexte politico-économique. C'est en supprimant ce qu'il y a à voir, le travail ouvrier, que le photographe impose une réflexion sur le travail et sur la perte de visibilité de celui-ci. Cette image de Philippe Bazin est extraite de cette série de vidéos, produite en 2002 lors d'une résidence d'artiste dans une entreprise privée écossaise. Les vidéos de Bazin fonctionnent comme des photographies étendues dans le temps. Le cadrage ne bouge pas, c'est un plan fixe, et la personne s'efforce elle aussi de rester statique face à la caméra. Ce dispositif n'est pas sans rappeler le dispositif photographique et le fameux mot du photographe : « on ne bouge plus!». On ne bouge plus, mais pas l'espace d'un centième de seconde: l'espace de soixante minutes. Le photographe étire ainsi le temps photographique et brouille les frontières entre photographie et vidéo.

La vidéo est donc constituée d'un seul plan séquence, immobile, montrant une personne fixant l'objectif, durant une heure. Une heure qui est une heure précise : la vidéo s'intitule Une heure de travail. L'image photo-vidéographique est alors mise en travail pour une heure, en même temps que le modèle, le sujet, la personne enregistrée, est mise au travail devant le dispositif de captation, pour une heure. Une heure prise sur son temps de travail : un temps de travail qui se trouve donc lui aussi détourné. Le travail réel des employés n'est pas de poser devant des dispositifs d'enregistrement: c'est le dispositif du photographe qui transforme leur temps de travail en temps de pose. Bazin prélève sur le temps de travail habituel une heure de travail détourné. On a donc un travailleur qui travaille une heure devant la caméra à regarder vers l'objectif. 
À regarder un spectateur qu'il ne voit pas, mais qui le regardera à son tour pendant une heure : une heure de temps de spectacle.

Le dispositif mis en place par le photographe reprend les codes de la photographiedocument: frontalité, éclairage neutre, netteté. Le photographe rejette toute esthétisation de l'image au profit de ses qualités descriptives. Mais le titre de la vidéo apparaît comme énigmatique dans la mesure où l'image ne montre pas le travail tel que nous avons l'habitude de le voir représenté. Cette disjonction, entre la monstration et sa légende, n'est pas anecdotique, mais bien plutôt constitutive de ses recherches et de sa manière de travailler. Le dispositif de la documentation tourne en quelque sorte à vide : les employés ne sont pas filmés dans les conditions qui permettraient l'étude sociologique, c'est-à-dire dans l'action du travail pour lequel ils apparaissent socialement comme employés. Le temps de travail dans Dufftown, c'est regarder face à une caméra. Le spectateur des images voit des hommes, des femmes qui le regardent pendant une heure. Le visage porte l'objet travail, sans pour autant que le travail ne soit visible. Renvoyé à son invisibilité, il est donc ce sur quoi porte l'interrogation : il est l'inconnu. Néantisant les représentations que nous avons du travail, le photographe nous renvoie à nos propres attentes face à l'image du travail. Ce que les vidéos d'une heure de travail construisent dans le négatif de ce travail que nous attendions et que nous ne voyons pas, c'est une autre forme de travail, un travail du regard. Le travail qui est mis là, concrètement en œuvre, c'est celui du sujet, une heure face à une caméra, du spectateur, une heure face à une vidéo. Le travail y apparaît être alors une question de regard, de celui que le sujet porte vers moi sans me voir directement, celui que je porte sur lui sans qu'il me voit. Le travail se constitue comme échange de regards qui se font dans la distance. Le travail n'y est plus un objet, une marchandise, mais une personne, deux personnes : lui et moi, là-bas et ici. Ce que la vidéo documente c'est donc le travail absent et ce travail qui met en œuvre un travailleur et un regardeur, un acteur et un consommateur. L'employé n'y est plus soumis au mode d'emploi de sa tâche, au travail habituel : il n'est plus ce qui est utilisé, mis au service de, ce qui est employé pour, l'anonyme qui s'efface derrière ce qu'il produit, il est acteur face à la caméra, acteur de sa présentation. De même, le spectateur ne peut être le consommateur du fruit du travail, mais celui qui, à l'autre bout de la chaîne productive, regarde en face celui qui travaille. Comment les deux regards s'ajustent l'un à l'autre est bien une question hautement politique puisqu'elle engage nos capacités, d'une part à se montrer, d'autre part à regarder, nos capacités à nous confronter, à ne pas oblitérer dans la production comme dans la consommation, la question de l'humain.

11 Les vidéos de Philippe Bazin inscrivent ainsi l'art, l'esthétique dans le souci de l'autre, et le souci de l'autre dans l'art. Les visages sont les pivots à partir desquels sont remises en cause nos habitudes de travail et de spectacle. Les vidéos de visage, temps de travail, temps de pose, font émerger le travail et sa représentation comme problème. L'enregistrement frontal des visages reprend les codes d'une imagerie de rationalisation du réel, le document clair et précis, en même temps qu'il met en échec la consommation esthétique de l'image. Philippe Bazin met ainsi les employés et les spectateurs au travail, à partir d'une image-carrefour. La photographie est alors un entremêlement, un tissage entre un espace-temps à distance et une épreuve du réel. Bazin cite les mots de Jean-François Chevrier qui parle de "documents d'expérience ». Le tissage consiste à articuler une politique de la distance et l'engagement dans le réel. Si l'appareil est effectivement médiation, la médiation ouvre sur un espace de création. Cette création ne vise pas à remplacer le réel par son savoir, ni à le passer au filtre des 
appareils pour le rendre manipulable, mais à tenter de faire exister une expérience pour le spectateur. Le tissage documentaire inclut d'emblée la question de la transmission: il est un projet en deux directions, vers le réel dont il souhaite apprendre, vers les spectateurs auxquels il souhaite montrer. Le retrait de l'auteur, telle que la forme finale l'impose au travers de la froideur des images, crée un espace d'accueil. Le photographe ne sature pas les images de son style et de sa vision, mais invite le spectateur sur le terrain de l'expérience neutre : c'est-à-dire d'une expérience qui n'est pas prémâchée pour lui, mais mise en problème, disponible pour son interprétation.

Photographie 5. Philippe Bazin, Des hommes en colère, 2010, 15 photogrammes.

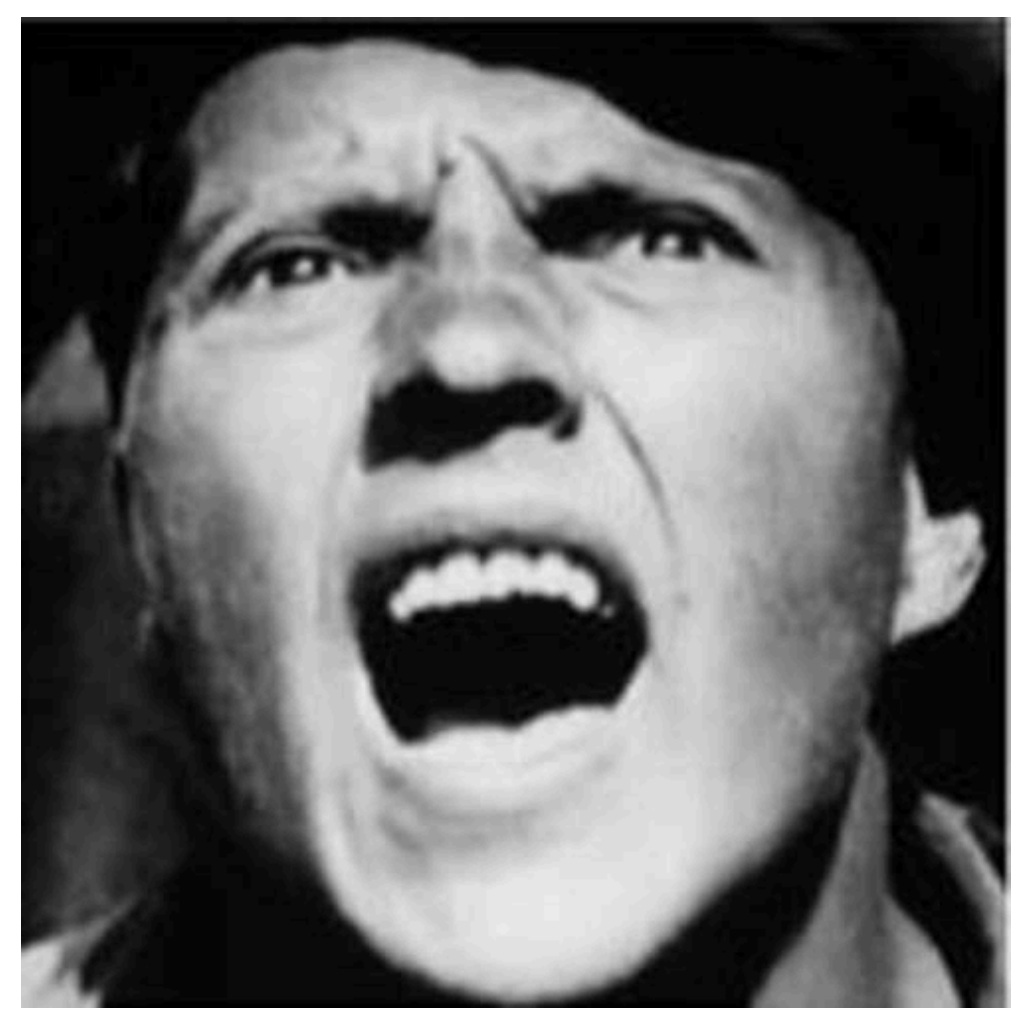

Dans une autre série, Des hommes en colère, Bazin construit un dispositif documentaire centré encore une fois sur les visages (Bazin, 2012, 73). Ces images constituent une série de 15 photogrammes extraits du film d'Eisenstein La Grève. La série est introduite par un propos de Maxime Strauch, ami et collaborateur d'Eisenstein: "Chaque visage entrevu devait apparaître comme une composante caractéristique de l'événement. L'homme n'étant montré que pendant un court instant, son aspect devait être excessif à l'extrême : son visage devait être, en quelque sorte, une philosophie, une conception du monde.» (Straub, 2002) Elle est accompagnée d'un extrait du texte de Christiane Vollaire intitulé " Désaffecter le politique ? Puissances de la colère. » (Vollaire 2010) Ce que Bazin opère, c'est un montage de deux textes avec un démontage du film d'Eisenstein. Là où les gros plans devaient jouer leur fonction expressive en un instant pour soutenir la force du montage dynamique d'Eisenstein, là où ils faisaient partie d'un tout, figures émergentes et coup de poing, ils font, dans la série de Bazin, l'entièreté du dispositif. Bazin ne produit pas un résumé de La Grève à partir de quelques photogrammes choisis, mais opère une coupe transversale qui met en lien ces 
visages. L'objet que la photographie documentaire se donne ici est un film. Elle ne constitue une critique du film qu'en élaborant une série d'images: cette critique ne doit-elle pas encore être entendue au sens de commentaire sur l'œuvre, mais d'exploration des réalités qu'elle draine. Bazin extrait du film des photogrammes aptes à dire quelque chose de cette fiction d'Eisenstein. La réduction du film aux visages est le parti pris documentaire de Bazin que nous devons à notre tour démonter, tenter de rendre. Remettre sur le devant de la scène, en présentant dans le champ de l'art contemporain des photogrammes du film d'Eisenstein réduisant celui-ci à la colère exprimée dans les visages, est une manière d'interroger notre capacité à lire aujourd'hui cette colère et à relier cette héritage à la situation contemporaine.

Bazin réduit le temps filmique au temps photographique. Cette réduction constitue un temps d'arrêt: c'est depuis cette "pause » que les images nous interrogent. Cette question du temps doit être envisagée pour elle-même car c'est un temps hautement politique que le film d'Eisenstein met initialement en œuvre. Tout d'abord La Grève est un film visant à saisir le spectateur, à le prendre dans une histoire et une émotion apte à lui apprendre à se révolter. Il se termine par ces mots : «Et des blessures inoubliables sur le corps du prolétariat ont laissé des traces à: Le Lena, La Talka, Zlatooust, Varoslav, Tsaritsyne, Kostroma. Ne l'oublie pas prolétaire!» (Eisenstein, 1925) Le temps qui se déroule dans la fiction est un métissage : temps de la fiction, il en appelle à un temps réel, temps de la révolution prolétarienne en marche. C'est un temps militant qui s'appuie sur le processus fictionnel pour agir dans le réel. La continuité du temps filmique est faite d'interruptions du récit par les gros plans dont les visages sont la principale source. Ils marquent des temps d'arrêt acméique dans le développement de l'histoire, temps d'arrêt qui opposent violemment le patronat au prolétariat avec le maillon intermédiaire que sont les délateurs et le pivot de l'action qui est le suicide de l'ouvrier accusé de vol. Les portraits figent les rapports de force pour opposer la posture des uns et des autres. Côté patronat, le rire gras, côté prolétaire on aura l'incompréhension et la colère. Les visages que retient Bazin sont ceux du prolétariat et le travail qu'il mène sur le film concerne l'expression de cette colère. C'est à la philosophie de cette colère exprimée dans les visages que le nouveau montage de Bazin convoque. C'est cette philosophie que Christiane Vollaire développe dans le texte qui accompagne les images. Le temps militant du montage d'Eisenstein passe dans un autre temps, celui de la pause qui impose de réfléchir, et demande au spectateur de l'interpréter.

14 Bazin ne met pas en dialogue ces images et les représentations du visage dans l'art du portrait : la clinicité de ses enregistrements ne visent pas l'art, mais la représentation de la colère, d'une colère contre la domination. Il est donc question de la position du sujet dans les images : non pas exemple-objet mais acteur-lutteur. On est chaque fois face à des individus. Des individus qui, dans le dispositif de Bazin, se passent de toute organisation extérieure, de tout appareil : des individus qui, comme dans la révolte des ouvriers, n'ont besoin d'aucune instance supérieure pour se retrouver, entrer en lutte. Comme Chris Marker se servait des images d'archives pour reparler, créer des ponts, qui sont une forme de raison, Bazin tisse un relai pour la colère: parce qu'effectivement la colère n'est pas une réponse purement émotionnelle, mais bien le mûrissement d'une révolte. La révolte comprend ce qui oppresse : n'a-t-elle pas les mots, elle en a les chemins intérieurs; n'a-t-elle pas les articulations logiques, elle en a l'analyse rationnelle. La colère est révolte contre la loi du plus fort qui fabrique 
l'oppression. La colère peut être reprise parce qu'elle peut être comprise : la colère a une base d'objectivité intrinsèque. Christiane Vollaire affirme avec justesse qu'elle est le signe de la démocratie: parce que celui qui est en colère travaille dans l'objet commun, la colère est toujours collective, vise toujours une organisation collective, quand bien même elle semble ne se porter que sur un individu. La colère mobilise de la communauté. Bazin reprend, remobilise. La stratégie du document qui occupe Bazin est de soustraire au flux des images des pièces à conviction: documents bruts d'un film, documents bruts représentant le projet d'Eisenstein et de toute une société, le projet d'Eisenstein au travers de ces visages en colère. Alain Montandon, dans l'ouvrage Les formes brèves, donne cette analyse du fragment: "[l]e fragment est défini comme le morceau d'une chose brisée, en éclats, et par extension le terme désigne une œuvre incomplète morcelée. Il y a, comme l'origine étymologique le confirme, brisure, et l'on pourrait parler de bris de clôture de texte. La fragmentation est d'abord une violence subie, une désagrégation intolérable. On a souvent répété que les mots latins de fragmen, de fragmentum viennent de frango: briser, rompre, fracasser, mettre en pièce, en poudre, en miettes, anéantir. En grec, c'est le Klasma, l'apoklasma, l'apospasma, de tiré violemment. Le spasmos vient de là : convulsion, attaque nerveuse, qui disloque.» (Montandon, 1992, 77) Si les visages, dans le montage d'Eisenstein, agissent comme une force qui oriente le déroulement et la lecture de l'action, séparés du reste des images par les fragments qu'en donne Bazin, ils sont déportés de ce rôle d'entraînement. C'est la structure philosophique qu'ils portent que les fragments cherchent à dégager de l'ensemble. Le visage est le lieu de l'expression violente, claire, manifeste : il est l'endroit de la manifestation. Par la fragmentation, Bazin rend visible cette conception de l'homme où le visage est jeu, masque, expression, où il porte sa vision du monde, sa manière d'être au monde. La continuité du film est brisée, son contexte idéologique aussi, mais ce que Bazin relève, prélève, c'est cette injonction de laisser être le visage comme liberté, comme interface d'un sujet et du réel, comme capacité à prendre un masque dans l'espace public. Il faudra donc rapprocher son travail du travail typologique d'August Sander, dans lequel visage, corps et façade vestimentaire, sont rendus à leur fonction sociale, renvoyés à leur place dans la société. L'œuvre de Bazin construit un passage entre La Grève et les Hommes du XXe siècle. Il reprend le principe d'isolement et de répétition documentaire que Sander met en œuvre pour enregistrer et donner à la comparaison les hommes en colère d'Eisenstein. La contemporanéité des deux artistes, Sander et Eisenstein, montre deux forces agissantes : le désir d'apprendre, de voir le réel tel qu'il est, et le désir de justice sociale, la volonté de réformes. Le projet de Sander passe par une description des classes sociales qui font la société allemande, description sans compromission, sans flatteries ; le projet d'Eisenstein passe par le militantisme, la création de l'œuvre révolutionnaire, engageant dans le même mouvement une révolution esthétique et politique. Le travail de Bazin tient en lui ces deux directions : à la fois les fragments donnent à penser l'œuvre, à la fois le montage du texte de Christiane Vollaire est une pensée d'un politique engagé, investi, affecté.

\section{Les Héritiers : une proposition documentaire sur l'école des ouvriers}

« le collège contribue à transformer les inégalités sociales devant la culture écrite en divisions 
scolaires, qui vont elles-mêmes préfigurer, préparer et renforcer les divisions de classe (...)

les sortants de l'enseignement professionnel étant voués pour la grande majorité d'entre eux à occuper, non seulement lors de leurs premières

années de vie active mais de plus en plus longtemps, des emplois d'ouvriers ou d'employés, c'est-à-dire des emplois relevant du salariat d'exécution. " Ugo Palheta ${ }^{3}$

Photographie 6 et 7. Auteur, Bac pro technicien de scierie, CAP Matelot, 2013.
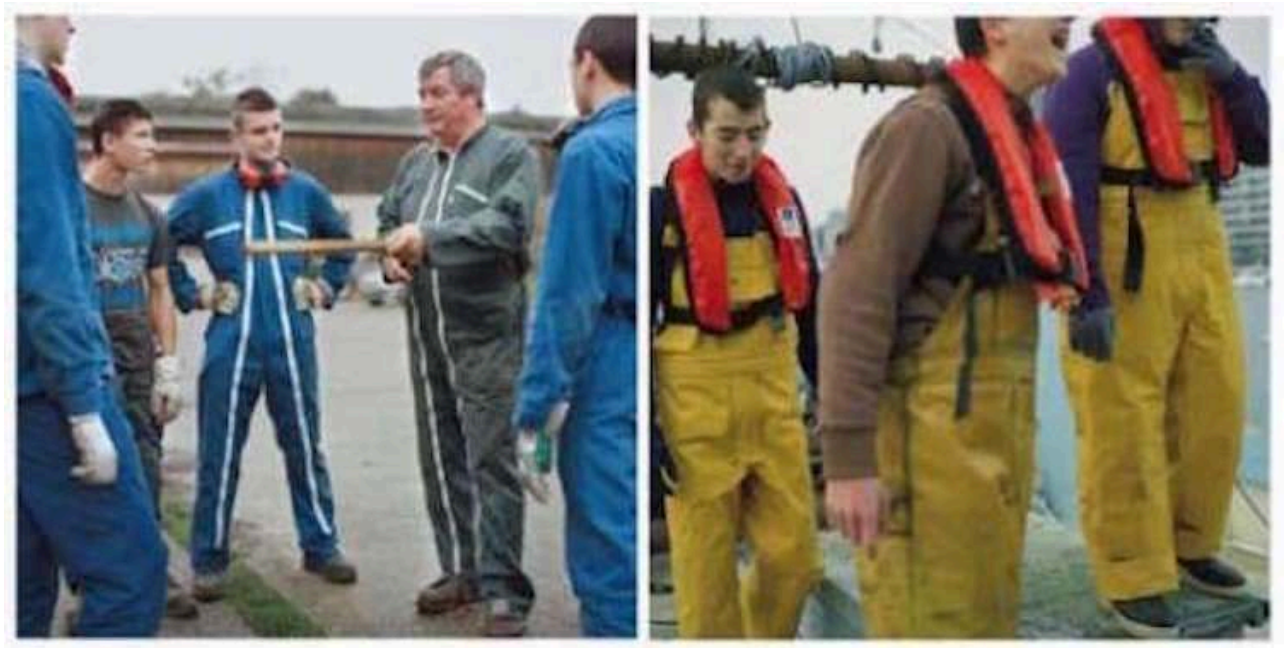

Le titre de la série qui est ici proposée reprend celui de l'ouvrage de Pierre Bourdieu et Jean-Claude Passeron (1964). Il est effectivement, dans cette série photographique, encore une fois, question du système scolaire, du lot de valeurs qu'il véhicule et de la part qu'il joue dans la reproduction des inégalités sociales. En revanche, les photographies prennent le contre-pied de l'ouvrage sur deux points au moins. D'une part, elles ne clivent pas les héritiers d'un côté et les classes populaires de l'autre, sousentendant que celles-ci seraient du côté des déshérités. Bien au contraire, les images montrent la richesse de l'héritage transmis aux étudiants en école professionnelle, héritage proprement positif. D'autre part, l'approche documentaire mise en œuvre s'éloigne du savoir sociologique et tente de favoriser une interaction dans l'élaboration des images. Les élèves se donnent à voir tout autant que la photographe donne sa manière de les voir.

La série ne s'inscrit donc pas du côté du savoir sociologique, mais du côté des jeunes, de l'expérience qu'ils font. Cette expérience ne peut être réduite à l'enquête sociologique. Bien qu'elle se situe indéniablement dans un contexte social, nous prenons le parti de la voir comme en excès par rapport à ce contexte. Si héritage social il y a réellement pour tout jeune entrant dans ce qu'on appelle la vie active, l'expérience que celui-ci fait de son environnement, de lui-même, appelle d'autres lectures. Elle appelle d'abord pour celui qui les prend en photo une empathie avec leur sentiment de l'existence, avec ces corps à corps dans lesquels ils s'éprouvent et éprouvent la réalité, corps à corps fait d'apprentissages techniques, de répétition de gestes et de postures, et aussi de jeux entre eux, de jeux avec son propre corps. S'ils sont les héritiers d'une position sociale 
qui détermine leur parcours et leur insertion professionnelle, leur manière de se percevoir, leurs espérances et leurs références symboliques, au même titre que les jeunes issus d'autres milieux socio-culturels, le parti pris photographique est d'être dans l'inconscience de ces déterminismes, d'être avec eux dans cette inconscience qu'a la jeunesse, qui éprouve et s'éprouve. Les photographies se veulent donc de terrain et non de savoir, d'empathie et non d'étude. Les formes du parcours initiatique qui feront définitivement appartenir l'individu à une société sont certes prégnantes. Il ne faut pourtant pas que ces méta-structures s'accaparent l'ensemble de l'expérience, qu'elles réduisent l'expérience à ce qu'elles peuvent en savoir et en dire. Cette ouverture au monde, cette plasticité des jeunes est ce qu'observe la série photographique Les héritiers, cette difficulté aussi à s'approprier les cadres culturels, les référents symboliques, qui font que les corps débordent, les regards s'éloignent, l'être se disperse. Les images, par leurs cadrages serrés, la proximité de l'appareil photographique avec ces corps au travail et en jeu, restituent quelque chose de ces tentatives dociles et des résistances rencontrées pour appartenir à un monde précis, pour réduire le champ des possibles à un parcours socio-professionnel. Les jeunes photographiés sont donc à la fois les héritiers, d'un capital social et culturel, tel que Bourdieu et Passeron l'ont fort justement analysé pour les étudiants en lettres, mais les héritiers problématiques et débordant les déterminismes.

Photographie 8. Auteur, Audrey, diplômée du bac pro agro-alimentaire, responsable qualité dans une usine de transformation de la viande, 2014.

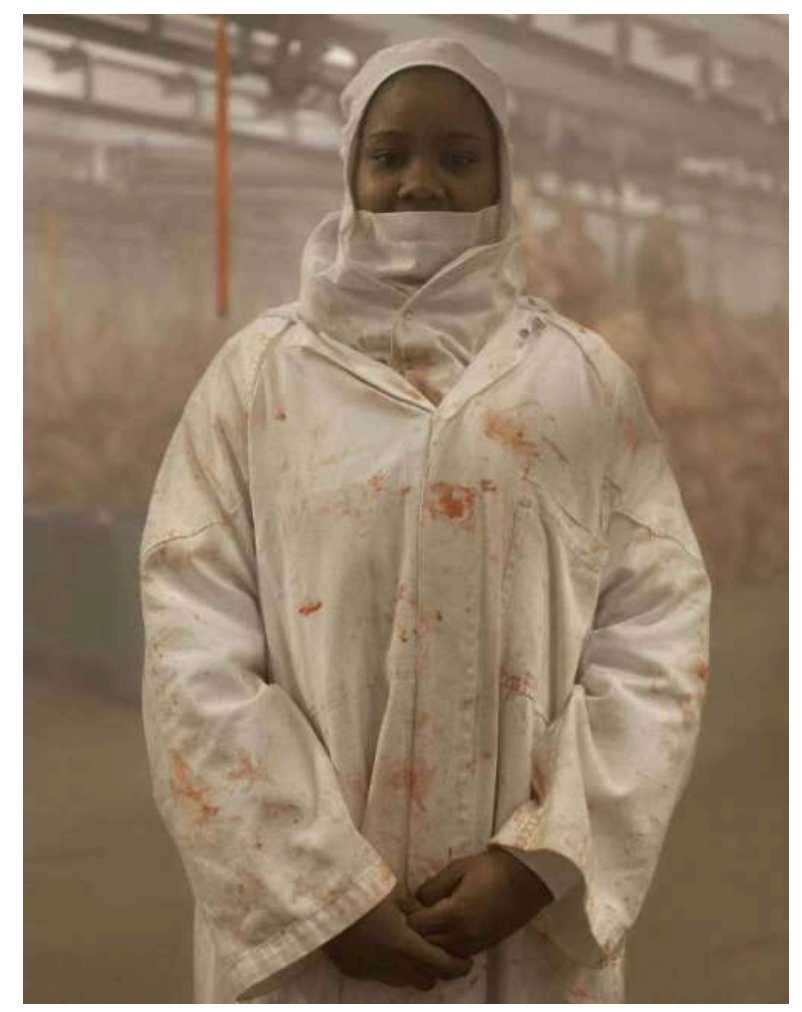

17 La série photographique engage ainsi autrement la notion d'héritage. Nommer une classe sociale, les déshérités, revient à supprimer l'héritage positif qui se transmet en réalité entre les individus. Si privation d'héritage il y a (car le terme déshérité indique bien une privation), elle est à penser dans le jeu des rapports sociaux : à qui, comment et par qui l'héritage est-il confisqué? La mauvaise réputation de l'enseignement 
professionnel pourrait être justement du côté de cette privation sociale de l'héritage et constituer un maillon à partir duquel le mépris des classes populaires et leur relégation économique et symbolique s'expriment. Les deux termes, d'enseignement et de professionnel, indiquent le jeu dans lequel l'enseignement professionnel est pris : d'un côté, le pouvoir des institutions et leurs objectifs souvent contradictoires, de l'autre le monde économique, ses attentes et son fonctionnement hiérarchisé. Le jeu du politique et de l'économique ne cesse de façonner, recréer et modeler les enseignements professionnels. Aussi, faire une histoire de ces écoles-là, c'est faire une histoire du tissu social dans lequel nous vivons. Comprendre l'héritage positif, c'est-à-dire actif, des écoles professionnelles sort les enseignements professionnels de leur enclavement, de leur fonction de rattrapage pour des enfants en échec scolaire.

Le travail documentaire a été réalisé en partie grâce à des dispositifs de résidences d'artiste en milieu scolaire mis en place par les Directions régionales des affaires culturelles. Ces longs séjours, la plupart du temps hébergés en internat, permettent de dépasser les frontières entre l'art, une culture souvent mal vue par les jeunes, et leur propre culture, mélange d'histoires sociale et familiale et de culture mainstream. Apprendre à se servir des moyens de représentation, à voir son monde sous un nouvel angle est un phénomène qui va dans les deux sens : les élèves découvre des techniques, une histoire visuelle et mettent en pratique leurs découvertes à l'épreuve de leur environnement scolaire; l'artiste développe ses propres moyens de production symbolique, et est remis en cause et interrogé par le monde qu'il découvre. Le travail documentaire n'est possible que parce que cette rencontre a lieu. Ensuite intervient la recherche des outils théoriques pour comprendre la réalité que nous façonnons ensemble, dans les distances qui nous séparent, sociale, culturelle, générationnelle, les blancs de la relation, les recherches de points communs, la curiosité réciproque. Et les questions qui apparaissent sont bien celles des opportunités offertes à cette jeunesse, de la réalité très concrète des formes d'enseignement et de leurs débouchés. Deux questions hantent la réalité visible qu'est leur vie à l'école : d'où viennent-ils, que deviennent-ils après l'école? Et progressivement se dessinent des parcours différents certes, mais régulièrement des répétitions où l'on peut lire la précarité sociale et familiale qui est le lot de nombreux d'entre eux. Les données sociologiques de l'ouvrage de Palheta sont éloquentes : la plupart des jeunes en filière pro sont issus des classes populaires (même s'il faut effectivement affiner ces catégories sociales). Les données sociologiques témoignent aussi des barrières sociales qu'ils rencontrent dans leur vie professionnelle. On se demande alors face à toute leur énergie ce que l'école fabrique. Et c'est une longue histoire qui se déroule, depuis cet après-guerre où l'école a en quelque sorte appartenu à la classe ouvrière, quand les anciens ouvriers devenaient professeurs d'atelier et transmettaient aux élèves leur culture de classe, jusqu'à aujourd'hui où les élèves se persuadent tout seuls qu'ils ne sont bons à rien, puisqu'ils sont là. Et l'on s'étonnera aussi de la séparation qui est faite entre le goût pour cet apprentissage, un certain goût du métier et la méfiance radicale à l'égard de ce qui les attend dans le monde du travail. On pourra penser qu'une certaine culture ouvrière, celle de la lutte et de la création d'un monde symbolique, persiste malgré tout. Mais l'usine qui emploie Audrey recrute ses ouvriers non qualifiés dans trois pays différents, avec des équipes logés dans des foyers, en rotation tous les quatre mois. Et l'on s'étonne de voir les enseignants la féliciter comme élève sage, et maintenant ouvrière modèle, en lui recommandant de bien travailler dans l'espoir d'un CDI. La valorisation du métier n'est certes pas attaquable, quoique les emplois occupés apparaissent parfois d'une 
ingratitude difficile à supporter. Mais les écoles professionnelles ne se sont-elles pas aussi construites sur une culture de la lutte sociale et de ses moyens de résistance ? La peur d'un avenir sombre pour les élèves, la mécompréhension de leurs attitudes de révolte et de provocation créent un climat scolaire plus propice à la survalorisation de la docilité, qui est un réel moyen d'apprentissage, qu'à l'affirmation de son corollaire, l'esprit critique et la défense de ses intérêts. Il faut aussi savoir que l'école pro s'est, d'une certaine manière, dé-professionnalisée: ses enseignants sont maintenant largement recrutés à la sortie même de leur formation ou quelques années après leur entrée sur le marché du travail. Ils sont donc davantage des spécialistes de la formation que d'anciens ouvriers. Le parti pris documentaire est de montrer la force de ces jeunes, l'intérêt des gestes professionnels et des apprentissages, de parler de cette histoire ouvrière dans sa relation à la question de la formation, et de mettre en question la docilité scolaire demandée entendue comme seule planche d'un salut social et professionnel.

\section{BIBLIOGRAPHIE}

Baetens J. (dir.), 2007, Critical Realism in Contemporary Art : Around Allan Sekula's Photography, Leuven, Leuven University Press.

Baqué D. (2009), Pour un nouvel art politique. De l'art contemporain au documentaire, Paris, Flammarion.

Baudelot C. et Establet R. (1975), L'école capitaliste en France, Paris, Maspéro.

Bazin P. (2006), Dufftown, Rouen, POC.

Bazin P. (2012), Face à faces, Paris, L'Harmattan.

Bazin P. (2012), Photographies et photographes, Paris, L'Harmattan.

Bourdieu P. et Passeron J.C. (2002 [1964]), Les héritiers, les étudiants et la culture, Paris, Les Éditions de Minuit.

Durand R. (2005), Croiser des mondes : Document 2, Paris, Jeu de Paume.

Eisenstein S. M. (2002 [1925]), La Grève, Films sans frontière.

Foster H. (2005), Retour du réel. Situations de l'avant-garde, Bruxelles, La lettre volée.

Lugon O. (2001), Le style documentaire d'August Sander à Walker Evans, Paris, Macula.

Maresca S. (1996), La photographie, un miroir des sciences humaines, Paris, L'Harmattan.

Montandon A. (1992), Les formes brèves, Paris, Hachette.

Noiriel G. ([1986] 2002), Les ouvriers dans la société française. XIXe - XXe siècle, Paris, Éditions du Seuil.

Palheta U. (2012), La domination scolaire. Sociologie de l'enseignement professionnel et de son public, Paris, PUF. 
Pelpel P. et Troger V. (2001), Histoire de l'enseignement technique, Paris L'Harmattan.

Pontbriand C. (dir.), 2012, Mutations. Perspectives sur la photographie, Göttingen, Steidl.

Saussier G. (2001) « Situations du reportage, actualité d'une alternative documentaire », Communications, vol. 71, p. 307-331.

Sekula A. (1995), Fish Story, Düsseldorf, Richter Verlag.

Sekula A. (2005), Le statut de l'auteur dans l'image documentaire : signature du neutre: Document 3, Paris, Jeu de Paume.

Sekula A. (2010), The forgotten space, DVD Wild Art Film.

Terral H. (2009), Éduquer les pauvres, former le peuple. Généalogie de l'enseignement professionnel français, Paris, L'Harmattan.

Vollaire C. (dir.), 2010, Écrits sur images. Sur Philippe Bazin, Paris, L’Harmattan.

Vollaire C. (2010), « Désaffecter le politique ? Puissances de la colère », Colloque international de théorie politique, La pensée et l'action dans le pouvoir. Colère : dynamiques soumissioninsoumisson et création politique, Lausanne, du 23 au 25 avril 2010.

Willis P. (2011 [1977]), L'école des ouvriers , Marseille, Agone.

\section{NOTES}

1. Allan Sekula, « Onze remarques et une question sur le documentaire », in Chantal Pontbriand, 2012.

2. Entretien avec l'artiste, in Thèse de doctorat, p. 419.

3. Ugo Palheta, 2012, p 81.

\section{RÉSUMÉS}

Le réalisme des images photographiques a livré au grand public comme au chercheur une matière féconde pour connaître et étudier le travail ouvrier. L'inscription de la photographie dans le champ artistique a donné naissance à un nouveau type de réalisme, que les photographes ont appelé le documentaire critique. Appliqué à la question ouvrière, celui-ci prend en charge de penser la situation actuelle du travail ouvrier, et non plus seulement de témoigner des événements. L'œuvre d'Allan Sekula propose une analyse globale du travail ouvrier dans le domaine maritime. Le photographe Philippe Bazin amène la représentation du travail ouvrier vers les enjeux de philosophie politique que pose la production capitaliste. L'intention d'interdisciplinarité de cette photographie, qui emprunte ses méthodes aux sciences humaines, à l'art et au journalisme, invite l'auteur de l'article à proposer ses propres recherches documentaires sur ce qu'est devenue l'école des ouvriers.

The photographic realism delivered to the public as to the researcher a fertile material to know and study the workers. The inclusion of photography in art created a new kind of realism, as photographers called the critical documentary. Applied to the labour question, it supports to 
think the current situation of the workers, and not just to represent the events. Allan Sekula 's work offers a comprehensive analysis of the workers in the maritime domain. Photographer Philippe Bazin introduces political philosophy questions with the representation of workers. The interdisciplinary intent of this photography, which borrows its methods to the humanities, art and journalism, invite the author of the article to present its own documentary research on what became the School of workers.

\section{INDEX}

Mots-clés : photographie, document, réalisme critique, transport maritime, école professionnelle

Keywords : photography, document, critical realism, shipping, trade school

\section{AUTEUR}

\section{GÉRALDINE MILLO}

Formée à la photographie à l'école supérieure Louis Lumière et Maître de conférence en théorie de l'art, Géraldine Millo mène des recherches plastiques et théoriques en photographie documentaire. Elle s'intéresse aux rapports entre l'individu et son environnement, architectural, social et professionnel. Ce qui l'intéresse est le point de jonction entre la vie privée de la personne, son intimité et les cadres sociaux auxquels elle participe : quartier, usine, entreprise, école... Depuis 2010, son travail porte sur la question des lycées professionnels et des centres d'apprentis en France. Elle participe ainsi à de nombreux dispositifs d'action culturelle en direction de ces jeunes. Ses images et ses articles régulièrement dans la presse, les revues scientifiques et les expositions. 